\title{
Culturally Responsive Practice for Indigenous Contexts: Provenance to Potential
}

Professor Letitia Fickel, University of Canterbury

Associate Professor Sonja Macfarlane, Waikato University

Professor Angus Macfarlane, University of Canterbury

Paper presented as part of the symposium on Global Teaching: Southern perspectives on working with diversity, at the European Conference on

Educational Research, Dublin Ireland, 22-26, 2016. 


\section{Whakatauki}

Ahakoa he iti, he pounamu

Although it is small, it is greenstone

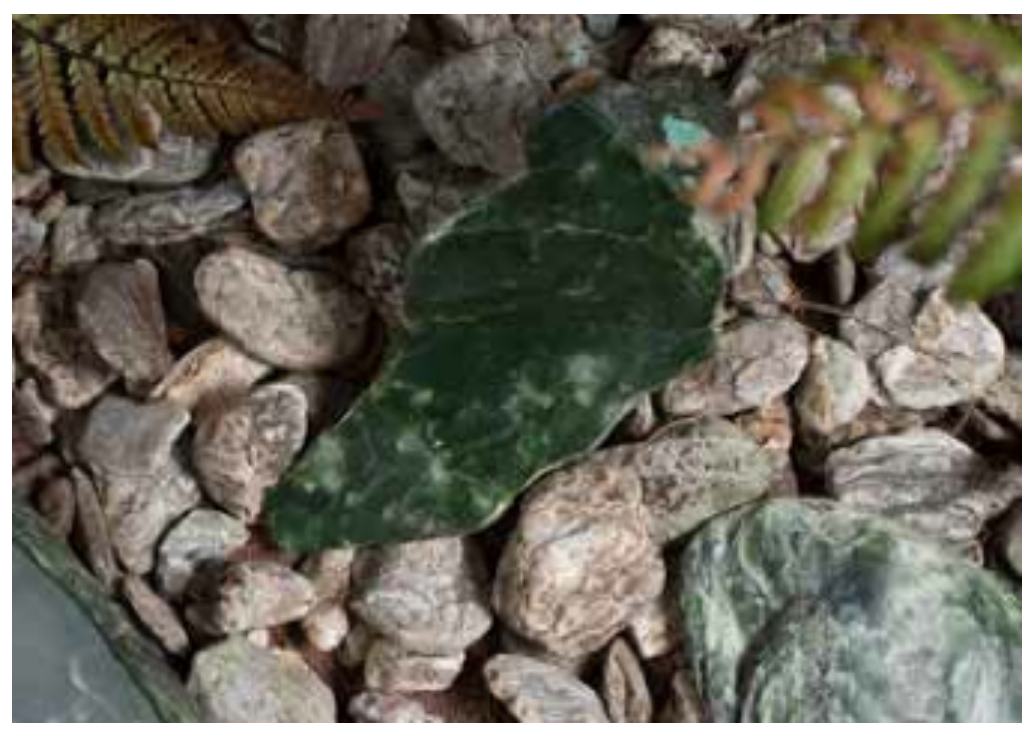




\section{Overview and Purpose}

- Historical and contemporary context of indigenous education

- Problematising culturally responsive practice (CRP)

- Lessons Learned from Practice: Alaska \& Aotearoa New Zealand TE Initiatives

- Reframing the conceptual and practice landscape of CRP 


\section{Context of Indigenous Education}

Historical

- Colonial history of compulsory schooling/boarding schools

- Explicit policies of cultural 'assimilationist'

- Native language 'eradication'

\section{Contemporary}

- High levels of non-attendance/drop-out rates

- Disproportional rate of expulsion/suspension from school

- Overrepresented in referrals to Special Education

- Disparity in educational outcomes/attainment 


\section{Culturally Responsive Practice}

Synthesis of literature suggests three key facets of cultural competence needed for teachers to engage in CRP:

- A constructivist understanding of knowledge, including both an epistemological stance of knowledge as dynamic, and a knowledgebase, or 'cultural literacy', that goes beyond the traditional Western canon.

- A sociocultural consciousness of self, including understanding both students and one's own culturally positioned attitude, beliefs, and experiences.

- The disposition and ability to engender caring, trusting, and respectful relationships with students and within the classroom among students. 


\section{Problematising Culturally Responsive Practice for Indigenous Contexts}

Castagno and Brayboy (2008) comprehensive literature review on culturally responsive schooling in the US context: "three topics that are rarely included in these discussions: sovereignty, racism, and epistemologies".

\section{Cultural Competence for} Indigenous contexts requires understanding of 5 sensitizing constructs:

- Self-determination and Sovereignty

- Settler Colonialism

- Racism and White Supremacy

- Language and Cultural Revitalization

- Indigenous Epistemologies 


\section{Lessons Learned from Practice: Alaska \& Aotearoa Teacher Education Initiatives}

Projects substaniated Indigenous self-determination and sovereignty placing Indigenous peoples' agency, aspirations and epistemology as the starting place.

-Indigenous educational and community members lead the theorizing and development of the PLD models, determined core practices, learning activities and knowledge-base

-Teacher 'cultural competence' was defined from a local, place-based perspective -Indigenous worldviews provided teachers with the critical lens to examine a different set of epistemological considerations of human development, health and well-being

-Teacher learning explicitly focused on the deconstruction of deficit theorizing of Indigenous culture, cultural practices and worldviews

-Non-Indigenous teachers engaged in direct experiences with cultural knowledge bearers/Elders to challenge teachers' assumptions of what 'competence' means and how it is enacted 


\section{Reframing the Conceptual and Practice Landscape of CRP}

\section{Māramatanga - Integrating new learning and knowledge}

The ability to articulate, integrate and apply Indigenous cultural knowledge and understanding in practice

1. To apply the intent /principles of Treaties and ensuing legal responsibilities with integrity in pedagogical practice

2. To actively ensure that equitable access to culturally meaningful and enhancing learning opportunities is contextually embedded for Indigenous learners

3. To access on-going and culturally appropriate mentorship (cultural advice, guidance and supervision) to ensure cultural competency and cultural safety

4. To pronounce the Indigenous language with integrity and authenticity, and incorporate it into all facets of learning and teaching activities, including engagement with the Indigenous community

5. To demonstrate the application and integration of Indigenous knowledge systems in pedagogical practice through the use of Indigenous approaches, frameworks, models, and programmes (ie: community consultation, curriculum content, programme planning, assessment approaches)

\section{Mātauranga - Exploring and Enhancing new learning and knowledge}

The ability to identify, interrogate and interact with Indigenous ways of knowing and doing: cultural knowledge, beliefs and values:

1. To understand the legal standing, impact and intent of Treaties and agreements on pedagogical practice

2. To understand the importance of creating culturally-affirming learning opportunities that enable all learners to walk in - and learn from - two worlds; Indigenous and mainstream

3. To identify one's own skill and knowledge gaps, and seek opportunities to undertake ongoing and purposeful professional learning and development

4. To address one's own learning needs specific to the use and pronunciation of the Indigenous language of that place

5. To explore and learn about culturally grounded Indigenous approaches, frameworks, models, and programmes developed from Indigenous knowledge systems

The ability to embrace and explore new learning and knowledge:

1. To understand the significant place of foundational Treaties and agreements to the self-determination and aspirations of Indigenous people

2. To understand how the impact of colonisation (ie: the loss of language, land, cultural practices, identity) has resulted in inequities / disparities for Indigenous learners in contemporary times

3. To have an awareness of one's own cultural identity, values, beliefs, practices, behaviours, and assumptions, and to consider one's identity within the local socio-historical context of that place

4. To respect the unique place of the Indigenous language of that place, and to view it as authentic and meaningful

5. To accept epistemological diversity: to acknowledge and reflect on cultural differences and similarities with an awareness that one's own cultural realities, perspectives, worldviews, approaches , knowledge systems, and frameworks may be different from others' 


\section{Quyana}
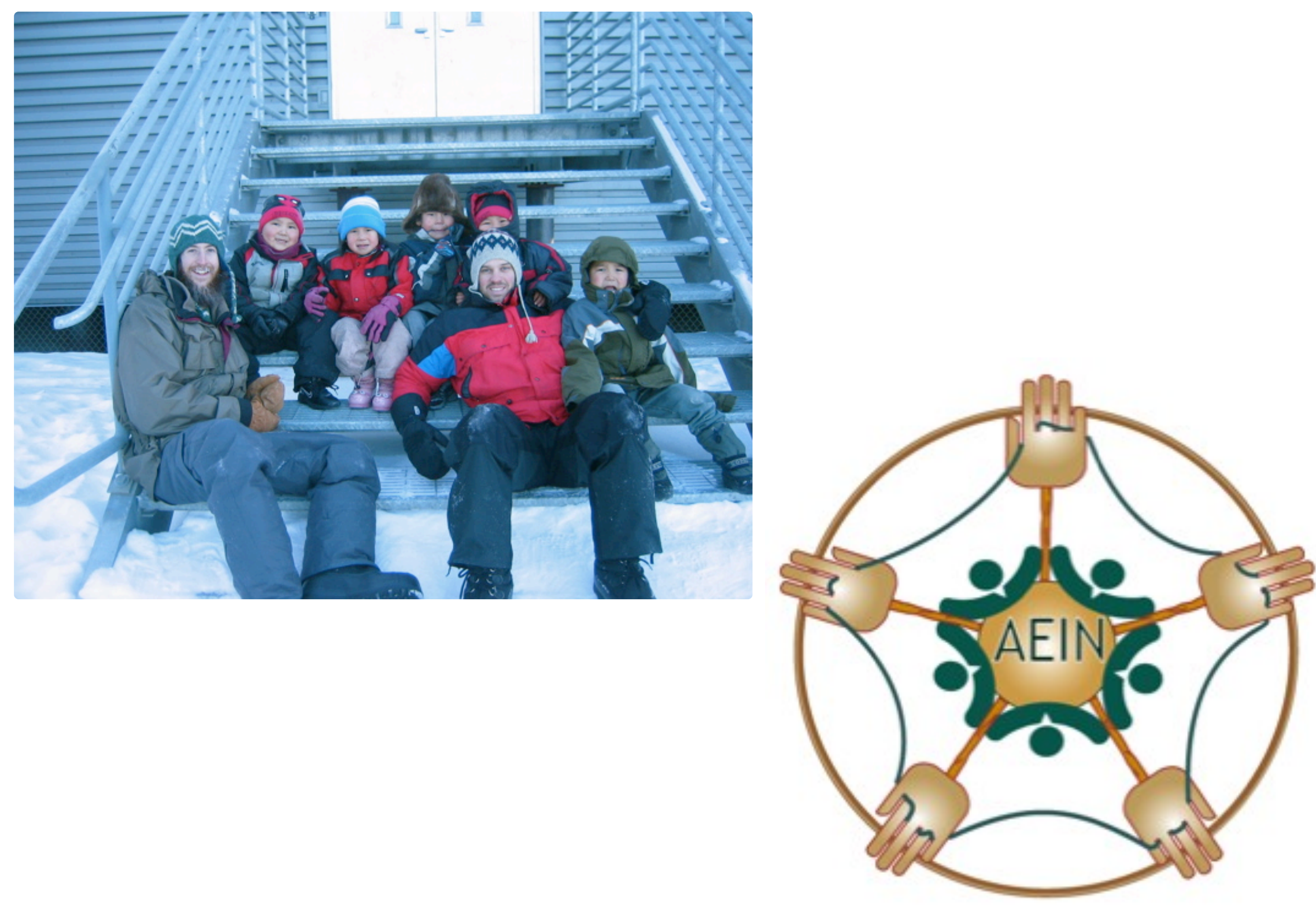